\title{
Isolated Hydatid Cyst in Retro-vesical Location with Unusual Presentation - A Case Report
}

Nazmun Nahar, S M Moinul Islam, Saiyeeda Mahmood, Gazi Abul Hossain, Nasim Khan, Ratan Kumar Chakraborty, Shakila Zaman Rima, Rawshan Ara and Fahima Akhter Dowel

Institute of Nuclear Medicine \& Allied Sciences, Mymensingh

Correspondence Address : Dr. Nazmun Nahar, Medical Officer, Institute of Nuclear Medicine \& Allied Sciences (INMAS), Mymensingh, BAEC, E-mail: nazmunshapla81@gmail.com

\begin{abstract}
Hydatid disease is caused by Echinococcus granulosus and is endemic in many parts of the worlds and produce cysts in almost every organs of the body, with the liver and lung being the most common sites. Here we presented a case of isolated retro-vesical hydatid cyst which is extremely rare with only a few cases reported in literature previously.
\end{abstract}

Key words: Hydatid cyst, Ultrasonography, CT scan.

\section{INTRODUCTION}

The hydatid cyst is a zoonotic disease caused by adult or larval stages of tapeworms belonging to the genus Echinococcus granulosus. Man is the incidental intermediate host where the definitive host is carnivores such as dogs. Larvae emerges from the eggs in the intestine; and after invasion to the blood vessels; they can migrate into almost every part of the body. The usual destination is the liver via the portal tract, but sometimes the larvae pass through the liver barrier and reach the lungs and all others viscera, where they transform into small cysts (1).The incidence of hydatid cysts constitutes in liver (59-75\%) followed in frequency by the lung (27\%). Involvement of the kidney $(3 \%)$, bone $(1-4 \%)$ and brain $(1-2 \%)$ is rare. Soft tissue, heart, spleen, pancreas and voluntary muscles, breast, thyroid, adrenal, ovary, bone and sub cutaneous tissue are unusual sites, but no site is immune $(2,3)$. The retro vesicle hydatid cyst is rare and constitutes only $6 \%-7 \%$ cases and it is considered to be an "aberrant" or "ectopic" site defined by the development of the parasite in the sub vesical and retrovesical fat (4).

\section{CASE REPORT}

A $55 \mathrm{yrs}$ old gentleman presented with the complaints of abdominal pain for 3 days and lump in the lower abdomen with frequency of micturation. On general examination - he was ill looking, mildly anemic, non icteric, rise of body temperature $(1010 \mathrm{~F})$ and normotensive. Per abdominal examination revealed mildly distended abdomen with lump in RIF region and tender RIF region. Investigations revealed her total count of WBC-14000/ cu mm of blood and differential count showed - Neutrophils-78\%, Lymphocyte-08\% , Eosinophil-12\% Monocyte- 02\% and Basophil -00\%, Hb-8.7 gm/dl, ESR-30 mm in 1st hour and Random blood sugar was $5.5 \mathrm{mmol} / \mathrm{L}$. He was sent for ultrasonography. Ultrasonography showed a cystic lesion posterior to the over distended bladder, compressing the bladder and bladder is slightly shifted towards right side. Both the kidneys showed mild hydronephrosis and inflamed appendix was seen in right iliac fossa region. Sonographic diagnosis was posterior bladder diverticulum. Then he sends for catheterization and after catheterization no change in the cyst size was seen. Then the patient sent for CT scan of abdomen for farther evaluation. Contrast enhanced CT scan of abdomen showed a large cystic lesion (>11.0 X $9.5 \mathrm{~cm})$ with thick enhancing wall in between the bladder and rectum in the retro-vesicle pouch and extending up to lumber region, displacing the rectum laterally (Figure 1), compressing the bladder anteriorly with slightly shifted towards right side (Figure 2). 


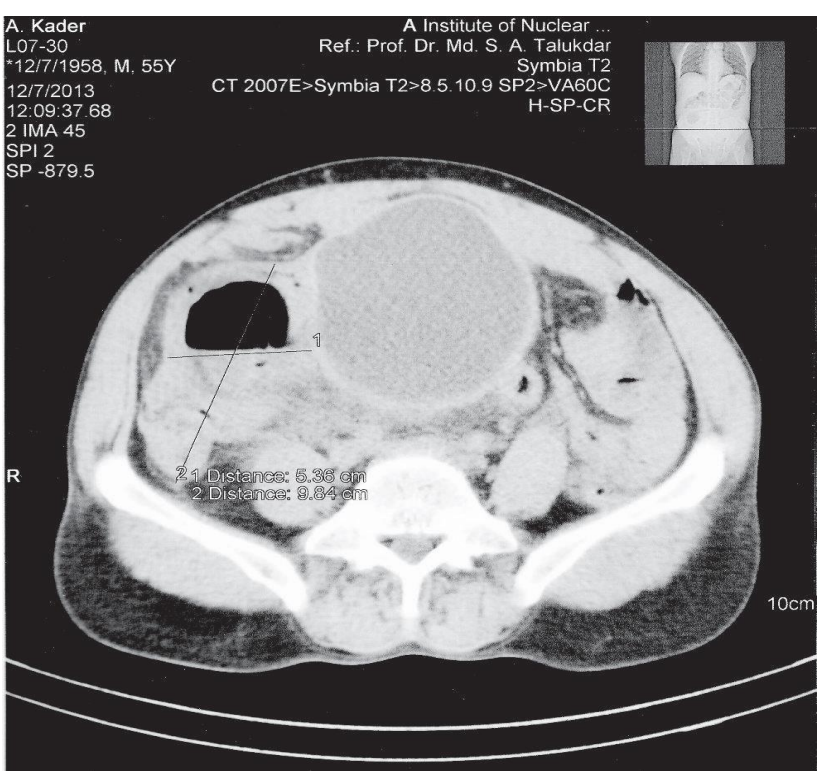

Figure 1: Axial image of CT scan of abdomen shows an appendicular abscess in the RIF region adjacent to the cystic lesion in the abdomen which shows thick enhancing wall.

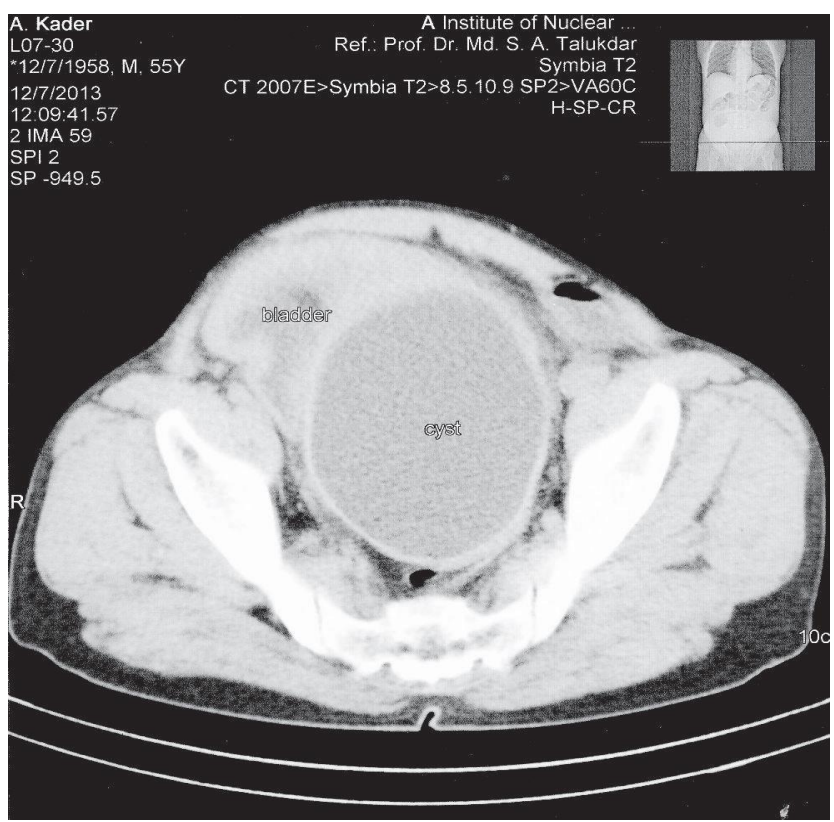

Figure 2: Axial CT scan of abdomen shows a thick walled cystic lesion posterior to the urinary bladder which apparently compressing the bladder and slightly shifted towards right side and gut loops upwards.

Gut loops were displaced superiorly by the cystic lesion. Sagittal \& coronal reconstruction images show a thick walled cystic lesion in the retrovesical location with about 10.4X $6.0 \mathrm{~cm}$ abscess formation with thickened gut wall is seen in right iliac fossa (RIF).Inflamed appendix with appendicolith was also seen in RIF region (Figure 3). Patient was undergone operative procedures and finally diagnosed as Hydatid cyst. The endocyst and ectocyst are come in close contact by the pressure effect of the cyst content and no daughter cysts are seen with the cyst. Histopathology confirms the diagnosis as Hydatid cyst. His post operative period is uneventful. Post operative follow up showed no recurrence of the cyst or any other intraabdominal pathology. On delayed follow up patient unluckily develop rectovesical fistula.

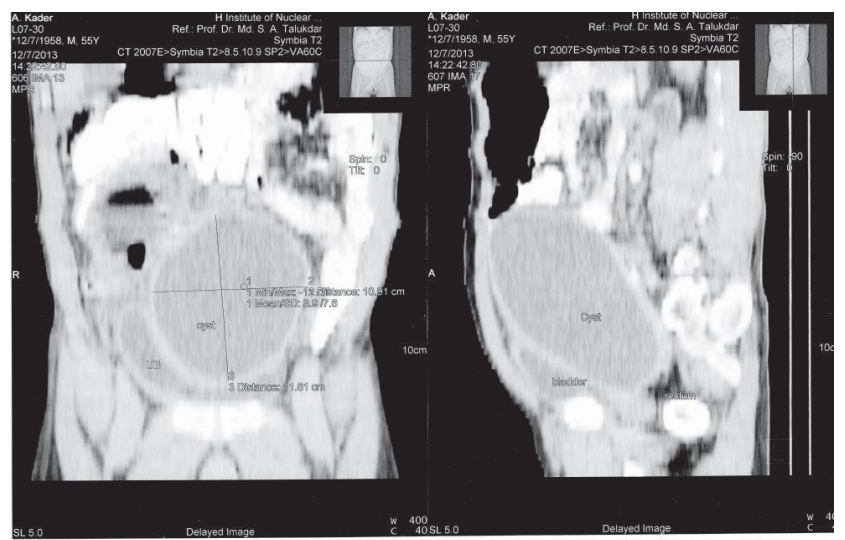

scan of abdomen show a thick walled cystic lesion posterior to the urinary bladder (retrovesical in location) in between the bladder and rectum with an adjacent appendicular abscess with thickened gut loops in RIF region.

\section{DISCUSSION}

Most hydatid cysts are asymptomatic and are diagnosed incidentally (2). The clinical manifestations in the hydatid cyst of most parts of the body are too non specific to make diagnosis based on sign and symptoms before surgery (1). A pathology based classification has recently been introduced by Lewall (5).The initial lesion (type I cyst) is a fluid- filled cystic structure with three layers: the outermost peri cyst composed of modified host cells and fibrous tissue; the middle laminated membrane resembling the white of an egg; and the thin inner germinal membrane. The germinal membrane produces the laminated membrane and the scoliosis that represent the larval stage (6). Hydatid cyst enlarged progressively, with the development of many daughter cysts within the cavity of the large parent cyst. 
The cystic fluid is a transudation of serum that contains proteins and is antigenic. When the type I cysts develop daughter cysts and/ or matrix they become type II cysts. When the nourishing Hydatid fluid is replace by formed elements the lesions dies and eventually calcified and biologically inert, forming the type III lesion (5) .

In uncomplicated cases of hydatid disease, the cyst content is clear as spring water, while infected cyst content is purulent fluid (7). Imaging modalities such as ultrasonography, CT scan and MRI have been the method of choice, especially in the later, which has been the diagnostic method of choice for the preoperative diagnosis of the hydatid cyst in most unusual location $(1,3)$. The characteristic imaging findings have been described as classification of cyst wall, the presence of daughter cysts or membrane detachment (8). However Radiologic signs are often non specific. Serological tests may be helpful in the diagnosis, but even their reliability is not $100 \%$ (9). Serology and imaging are the main tools for establishing diagnosis. Ultrasound is the preferred firstline imaging, but CECT give more precise information regarding the morphology (size, location, neighbor hood, and number) of the cyst (10). Magnetic Resonance Imaging is particularly useful because of its multiplaner capabilities and more detail anatomical resolution. Plain radiography is useful in certained circumstances for example imaging pulmonary and bone lesions as well as calcification in soft tissues. The unusual sites of this disease can frequently causes diagnostic problems and lead to diagnostic delays and many potentially serious complications. Peritoneal hydatid disease could be primary or more frequently secondary to rupture of hydatid cyst in liver or rarely in spleen. The primary peritoneal Hydatid disease is rare and has been reported to occur only in $2 \%$ of all abdominal Hydatid disease cases. The possible pathogenesis of isolated retrovesical hydatid cyst is that a small primary focus in the liver may rupture and seeds it contents into the pelvis and then the primary focus may undergo spontaneous resolution (11). On imaging retrovesical Hydatid cyst may mimic the following conditions: rectal duplication cyst, rectovesical neoplasm, posterior bladder diverticulum, cyst of the seminal vesicle, hydronephrosed pelvic kidney and a large ectopic ureterocele. The presence of daughter cyst may pathognomonic. Our patient present had a simple cyst with thick wall on imaging modalities. In some cases the final diagnosis may be made after surgery (12). The retrovesical site of hydatid cyst is rare and treatment is essentially surgical (4).

\section{REFERENCES}

1. B Geramizadeh. Unusual Locations of the Hydatid Cyst: A Review from Iran. J Med Sci 2013; 38(1) 2-14.

2. M H Dahniya, RM Hanna, S Ashebu, S A Muhtaseb, A ElBeltagi, S Badr, E El-Saghir. The Imaging Appearances of Hydatid Disease at Some Unusual Sites. The British Journal of Radiology 2001;74: 283-289.

3. N Bal, N E Kocer, R Arpaci, A Ezer, F Kayaselcuk. Uncommon Locations of Hydatid Cyst. Saudi Med J 2008; 29 (7): 1004-8.

4. M K Khouaja, N B Sorba, N Haddad, AT Mosbah. Retrovesical Hydatid Cyst: Diagnosis and Treatment in 8 Cases. Prog Urol 2004; 14 (4): 489-92.

5. D B Lewall. Hydatid Disease: Biology, Pathology, Imaging and Classification. Clin Radiol 1998; 52: 863-84.

6. I Beggs. The Radiology of Hydatid Disease. AJR Am J Roentgenol 1985; 145:639-48.

7. F Arsalan., K Zengin, A R Ozaras,. and F Tabak. Pelvic and Retroperitoneal Hydatid Cyst Superinfected with Brucella sp. And Review of Infected Hydatid Cysts. Tropical Biomedicine 2013; 30(1):92-96

8. I S Pedrosa , A Saiz, J Arrazola, J Ferreiros, and C S Pedrosa Hydatid Disease: Radiographic and Pathologic Features and Complications. Radiographics 2000; 20 (3)795-817

9. S I Beggs. The Radiology of Hydatid Disease. The American J of Roentgenology 1985; 145(3): 639-48.

10. Q F Parry, N S Wani, S Bazaz, R Khan, S N Malik. Primary Pelvic Hydatid cyst- A Case Report. Case Report in Surgery 2011 article ID: 809387.3 pages.

11. J C Angulo, J Escribano, A Diego, Sanchez-Chapado. Isolated Retrovesical and Extrarenal Retroperitoneal Hydatidosis: Clinical Study of 10 Cases and Literature Review. J Urol 1998; 159: 76-82

12. Dogra, Prem, Javali, Tarun, Saini, Ashish, Sharma, Sanjay, Gupta, Narmada. Case Report- Isolated Retrovesicle Hydatid cyst, BJU international. DOI: 10.1002/BJUIw-2010-019-web. 